Proceedings of the International School and Conference on Optics and Optical Materials, ISCOM07, Belgrade, Serbia, September 3-7, 2007

\title{
Fluorescence Spectra Study in Extremely Thin Cs-Vapor Layers
}

\author{
K. Vaseva ${ }^{a, *}, \mathrm{P} . \mathrm{Todorov}^{a}, \mathrm{D} \cdot \mathrm{Slavov}^{a}, \mathrm{~S} \mathrm{Cartaleva}^{a}$, \\ K. KOYNOV ${ }^{b}$ AND S. SALtiel ${ }^{b}$ \\ ${ }^{a}$ Institute of Electronics, Bulgarian Academy of Sciences \\ 72 Tzarigradsko Shosse bld, 1784 Sofia, Bulgaria \\ ${ }^{b}$ Sofia University, Faculty of Physics, 5 J. Bourchier bld, 1164 Sofia, Bulgaria
}

\begin{abstract}
The high resolution spectroscopy of nanometric-thin alkali-vapor layers was made possible through the development of extremely thin cell. We have investigated the behavior of the extremely thin cell fluorescence spectra both experimentally and theoretically. Experiment is performed on the $D_{2}$ line of Cs-vapor-layer with thickness $L=m \lambda$, where $m=0.5,0.75,1,1.25$. For open optical transitions, the fluorescence spectra shows narrow dip on the top of the sub-Doppler-width fluorescence profile, at $L \geq \lambda$. In case of closed transition, an extremely small feature in the fluorescence slightly shifted from its maximum is observed at $L=(5 / 4) \lambda$. The used theoretical model, based on the optical Bloch equations is in qualitative agreement with the experimental observations.
\end{abstract}

PACS numbers: 32.30.-r, 32.70.Jz

\section{Introduction}

High resolution laser spectroscopy of atoms confined in a thin cell $(10-1000 \mu \mathrm{m})$ is promising for the investigation of complex spectra of atoms and molecules $[1,2]$. Further study of alkali-vapor layers has been made possible through the development of extremely thin cells (ETC) (of thickness $\leq 1 \mu \mathrm{m}$ ) [3]. The vapor-layer thickness can be precisely controlled in an interval around the wavelength of the irradiating laser light. Interesting coherent effects are observed in absorption profiles changing their width periodically, with minima at $L=(2 n+1) \lambda / 2(n$ - integer $)[4]$. At the same time similar periodicity has not been reported in relation to the fluorescence and less attention has been paid to the fluorescence profile behaviour.

*corresponding author; e-mail: kapkavaseva@ie.bas.bg 
Here we present a detailed comparison of experimental and theoretical profiles of the fluorescence on the $D_{2}$ line of Cs-atomic layers with thickness $L=m \lambda$ (where $m=0.5,0.75,1,1.25$ ), when irradiated by frequency tunable singlefrequency diode laser light.

\section{Experimental investigation of the fluorescence spectra}

The experimental setup containing as radiation source a cw extended cavity diode laser (ECDL), operating in single-frequency mode with $\lambda=852 \mathrm{~nm}$ and linewidth of about $3 \mathrm{MHz}$ is shown in Fig. 1. The main part of the laser light is sent normal to the ETC and the fluorescence is measured directly by photodiode PD1. For this experiment, an improved registration of the fluorescence is elaborated that allows observation of small features non-reported in [5]. The second part of the laser light is sent to a scanning Fabry-Perot interferometer for monitoring the single-mode operation of the laser. The last part goes to $3 \mathrm{~cm}$ long Cs cell, for registration of saturated absorption ( $\mathrm{SA}$ ) spectrum needed for precise frequency reference and scaling for the DL frequency tuning. The laser light is linearly polarized and its frequency is scanned in the region of the hyperfine (hf) transitions starting from ground-state level with quantum number $F_{\mathrm{g}}=4$, on the $D_{2}$ line of Cs. The sub-Doppler spectra in the fluorescence are recorded for different laser light intensities and cell widths.

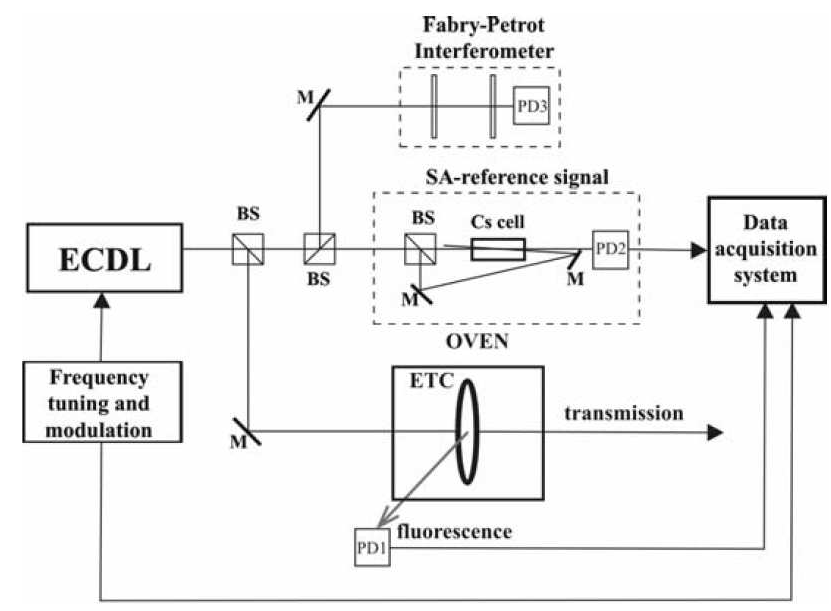

Fig. 1. Experimental setup.

In Fig. 2a, the fluorescence spectra of the hf $6 S_{1 / 2}\left(F_{\mathrm{g}}=4\right) \rightarrow 6 P_{3 / 2}\left(F_{\mathrm{e}}=3\right.$, $4,5)$ transitions are presented, for four different irradiating powers and $L=\lambda / 2$. Due to the small cell width, atoms with velocity normal to the cell windows give very small contribution to the fluorescence signal compared to atoms flying parallel to the ETC windows, which have larger time of interaction with the laser beam. 


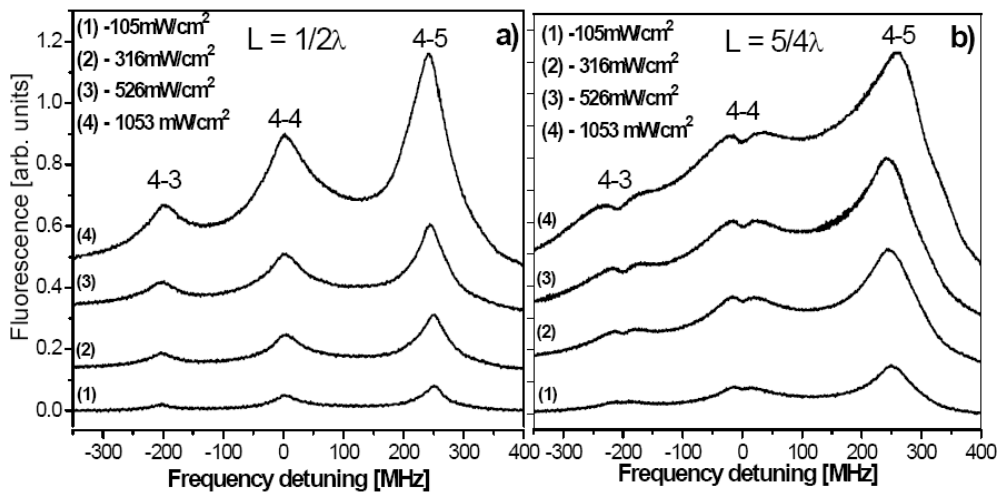

Fig. 2. Fluorescence spectra for $L=\lambda / 2$ (a) and $L=(5 / 4) \lambda$ (b) (frequency detuning: from $F_{\mathrm{g}}=4 \rightarrow F_{\mathrm{e}}=4$ transition).
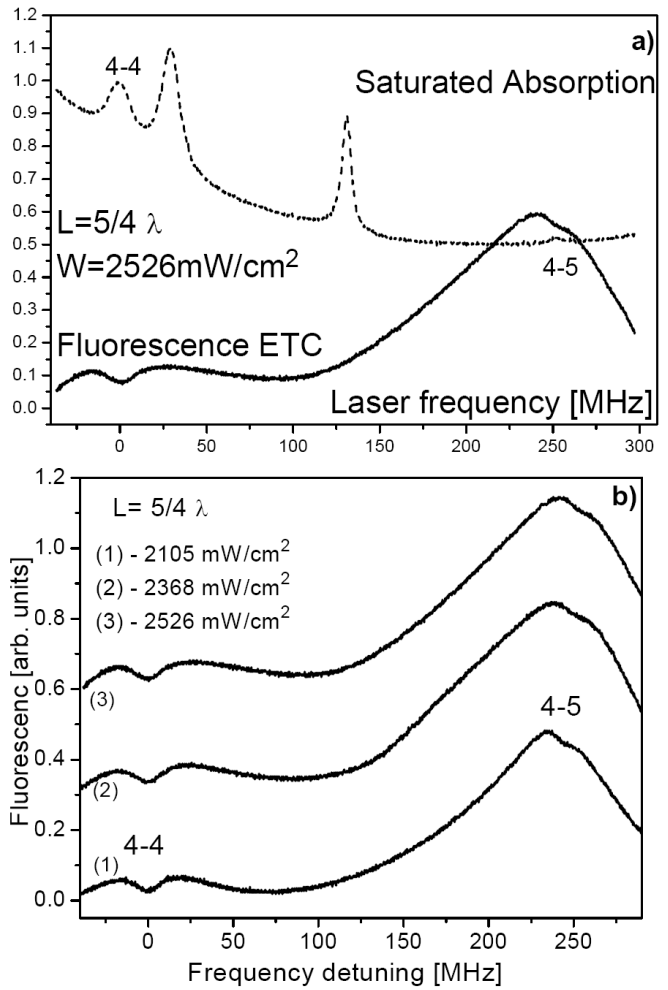

Fig. 3. Fluorescence spectra around $F_{\mathrm{g}}=4 \rightarrow F_{\mathrm{e}}=4,5$ transitions, for $L=(5 / 4) \lambda$ : (a) including SA reference signal and (b) for three different laser powers.

In agreement with previous investigations [3-5] we observe that the individual hf transitions are very well resolved. The reason is that the fluorescence signal comes mainly from slow atoms, whose velocity is small enough to allow time for 
absorption of a photon and subsequent spontaneous emission before collisions with the cell wall. In Fig. $2 \mathrm{~b}$, the results for cell thickness $L=(5 / 4) \lambda$ are presented. Here, similar to the results reported in [5] (at $L=\lambda$ ), the open $F_{\mathrm{g}}=4 \rightarrow F_{\mathrm{e}}=3,4$ transitions start to undergo optical pumping leading to reduced-fluorescence dips at the centre of hf transitions. The closed $F_{\mathrm{g}}=4 \rightarrow F_{\mathrm{e}}=5$ transition does not show any dip in the fluorescence at low laser intensities. However, increasing the power an extremely small feature is formed at the centrE of the hf transition.

To see better the mentioned feature, a zoom of the $F_{\mathrm{g}}=4 \rightarrow F_{\mathrm{e}}=4,5$ transitions for $L=(5 / 4) \lambda$ is shown in Fig. 3. The centre of the closed transition is shifted to the right from the maximum of the fluorescence profile, as one can see from the SA reference signal (Fig. 3a). Hence, in addition to the previous results [5] our experiment shows that in the fluorescence profile of the closed transition there is also some peculiarity although much less pronounced than that at the open transitions and of different shape. The appearance of similar feature was reported in [6] but for much larger cell thickness $(L \geq 2 \lambda)$.

We have also investigated the cases for $L=(3 / 4) \lambda$ and $L=\lambda$. For $L=$ (3/4) $\lambda$, the fluorescence behaviour is similar to that at $L=\lambda / 2$ at low power but with the power enhancement small modification of the tops of the fluorescence of the open transitions is observed. For the open transitions, the behaviour of the fluorescence signal at $L=\lambda$ is in agreement with that reported in [5]. Taking advantage from the improved registration, an extremely small modification of the fluorescence top at the closed transition (smaller than that observed at $L=(5 / 4) \lambda$ ) is already seen for $L=\lambda$ at high light power.

\section{Theoretical investigation and comparison with the experimental results}

To analyse the experimental results, we use the theoretical model presented in [5] and based on the optical Bloch equations. Simulations of the fluorescence profiles result in qualitative agreement between the theory and the experiment. The calculations are made for two-level system, closed or open transitions, as well with parameters applicable to an elementary and realistic case. We used the following parameters: $\gamma_{21}=5 \mathrm{MHz}, k u=250 \mathrm{MHz}$, and $\alpha=1$ (closed system) or $\alpha=0.5$ for a realistic open alkali system. Here $\gamma_{21}$ is half of the transition rate of the excited state, $k u$ is the most probable Doppler shift of the emitted light and $\alpha$ is the probability to decay from level 2 to level 1 .

At $L=(5 / 4) \lambda$, the calculated fluorescence spectra show a narrow dip superimposed on the top of the sub-Doppler-width fluorescence profile (Fig. 4a), for the open transitions suffering loss due to hyperfine or/and Zeeman optical pumping. For the closed transitions (Fig. 4b), with increasing power at first some "plateau" is formed on the top of the fluorescence profile and later it is modified in an extremely small peak that is superimposed on a very broad dip. Hence, the structure that appears on the top of the fluorescence of the closed transition differs significantly from the fluorescence dip occurring in the case of the open transition. The 

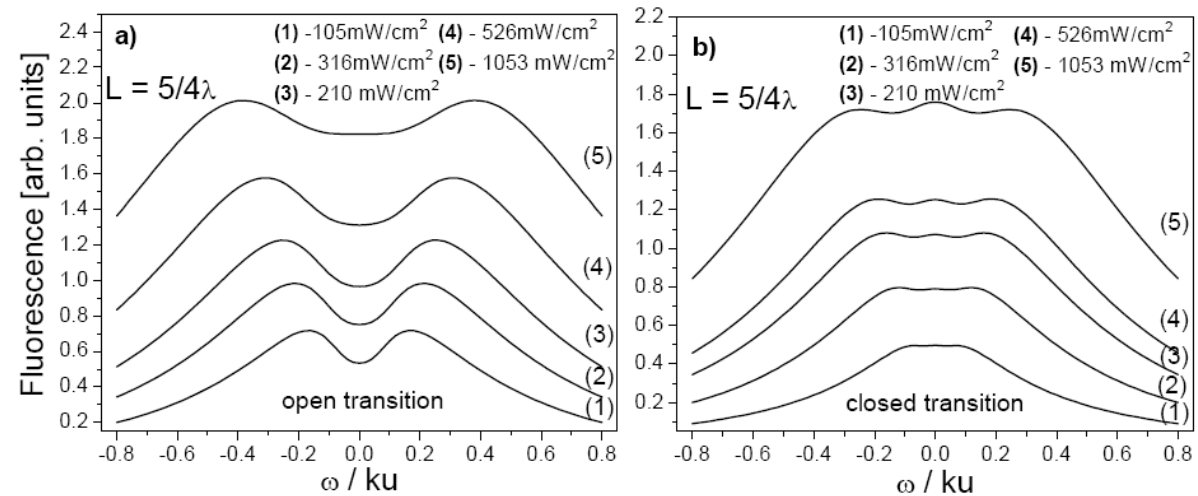

Fig. 4. Theoretical calculations of the fluorescence spectra for open (a) and closed (b) transition at $L=(5 / 4) \lambda$.

reason for this difference can be attributed to the fact that the closed transition is affected mainly by the saturation in absorption, while for the open one the loss due to the optical pumping is dominating.

\section{Conclusion}

We have studied the fluorescence spectra of Cs atoms confined in ETC as a function of the light intensity, at different cell thicknesses. Well pronounced narrow dip in the fluorescence (superimposed on the top of the sub-Doppler-width fluorescence profile) is observed experimentally only for the open transitions suffering hyperfine/Zeeman optical pumping and $L>\lambda / 2$. In the case of closed transition (non-suffering population loss), an extremely small feature in the fluorescence profile is observed better pronounced at $L=(5 / 4) \lambda$. For a detailed study of this feature, an ETC with thickness up to $2 \lambda$ would be helpful. However, such thickness is not possible to be approached with the available ETC.

The presented results contribute to the further advancement in the fundamental studies of saturation and optical pumping in extremely thin vapour layers. They show that the saturation of the fluorescence with light power strongly depends on the population loss of the two-level system.

\section{Acknowledgments}

Authors are grateful to Prof. D. Sarkisyan for providing the ETC as well as to INTAS (grant: 06-1000017-9001), B.F.S.R. (grant F-1404/04) and the French-Bulgarian Rila collaboration (French grant: 98013UK, Bulgarian grant: 3/10) for the partial supports of the work. 


\section{References}

[1] S. Briaudeau, D. Bloch, M. Ducloy, Europhys. Lett. 35, 337 (1996).

[2] S. Briaudeau, S. Saltiel, G. Nienhuis, D. Bloch, M. Ducloy, Phys. Rev. A 57, R3169 (1998).

[3] D. Sarkisyan, D. Bloch, A. Papoyan, M. Ducloy, Opt. Commun. 200, 201 (2001).

[4] D. Sarkisyan, T. Varzhapetyan, A. Sarkisyan, Yu. Malakyan, A. Papoyan, A. Lezama, D. Bloch, M. Ducloy, Phys. Rev. A 69, 065802 (2004).

[5] C. Andreeva, S. Cartaleva, L. Petrov, S.M. Saltiel, D. Sarkisyan, T. Varzhapetyan, D. Bloch, M. Ducloy, Phys. Rev. A 76, 013837 (2007).

[6] S. Cartaleva, K. Koynov, S. Saltiel, D. Sarkisyan, D. Slavov, P. Todorov, K. Vaseva, in: Proc. 34th EPS CPP, 2-6 July Warsaw (Poland) 200\%, accepted for publication. 